\title{
Immunogenicity and safety of mRNA COVID-19 vaccines in people with multiple sclerosis treated with different disease-modifying therapies
}

\author{
Fioravante Capone ${ }^{1}(1) \cdot$ Matteo Lucchini ${ }^{2,3}$. Elisabetta Ferraro ${ }^{4}$. Assunta Bianco ${ }^{2,3}$. Mariagrazia Rossi ${ }^{1}$. \\ Alessandra $\mathrm{Cicia}^{2,3} \cdot$ Antonio Cortese $^{4} \cdot$ Alessandro Cruciani $^{1} \cdot$ Valeria De Arcangelis ${ }^{2} \cdot$ Laura De Giglio $^{4}$. \\ Francesco Motolese ${ }^{1} \cdot$ Biagio Sancetta ${ }^{1} \cdot$ Massimiliano Mirabella ${ }^{2,3} \cdot$ Vincenzo Di Lazzaro $^{1}$
}

Accepted: 17 November 2021 / Published online: 3 December 2021

(c) The American Society for Experimental NeuroTherapeutics, Inc. 2021

\begin{abstract}
The potential impact of disease-modifying therapies (DMTs) for multiple sclerosis (MS) on COVID-19 vaccination is poorly understood. According to recent observations, the humoral immune response could be impaired in patients treated with ocrelizumab or fingolimod. Our study evaluated the immunogenicity and safety of mRNA COVID-19 vaccines in a convenience sample of 140 MS patients treated with different DMTs, undergoing vaccination between April and June 2021. Humoral immune response was tested 1 month after the second dose, using a chemiluminescent microparticle immunoassay to detect IgG against SARS-CoV-2 nucleoprotein. We explored the potential correlation between the IgG titer and DMTs. All patients in treatment with first-line DMTs, natalizumab, cladribine, and alemtuzumab, developed a measurable humoral response. In patients treated with ocrelizumab and fingolimod, the IgG level was significantly lower, but only some patients $(22.2 \%$ for fingolimod and $66 \%$ for ocrelizumab) failed to develop a measurable humoral response. In the ocrelizumab group, the IgG level was positively correlated with the time from last infusion. No SARS-CoV-2 infections were reported after vaccination. The most reported side effects were pain at the injection site (57.1\%) and fatigue (37.9\%). No patient experienced severe side effects requiring hospitalization. Our study confirms that COVID-19 vaccination is safe and well-tolerated in MS patients and should be recommended to all patients regardless of their current DMTs. Since fingolimod and ocrelizumab could reduce the humoral immune response, in patients treated with these drugs, detecting SARS-CoV-2 antibodies could be helpful to monitor the immune response after vaccination.
\end{abstract}

Keywords Multiple sclerosis · Disease-modifying therapies · COVID-19 $\cdot$ Vaccination · Humoral response · Immunogenicity

Massimiliano Mirabella and Vincenzo Di Lazzaro equally contributed.

Fioravante Capone

f.capone@unicampus.it

1 Neurology, Neurophysiology and Neurobiology Unit, Department of Medicine, Università Campus Bio-Medico di Roma, Via Alvaro del Portillo 200, Rome 00128, Italy

2 UOC Neurologia, Fondazione Policlinico Universitario Agostino Gemelli IRCCS, Rome, Italy

3 Dipartimento di Neuroscienze, CERSM, Università Cattolica del Sacro Cuore, Rome, Italy

4 Multiple Sclerosis Centre, S. Filippo Neri Hospital, Rome, Italy

\section{Introduction}

Severe acute respiratory syndrome coronavirus type-2 (SARS- CoV-2) is the causative agent of coronavirus infectious disease 2019 (COVID-19). This complex and multisystemic syndrome has spread around the world starting in November 2019 affecting, to date, $176 \mathrm{mln}$ of people, with $3.8 \mathrm{mln}$ of death [1].

Although innovative therapeutic protocols were introduced to support COVID-19 patients, leading to a significant reduction in death and severe complications, the real breakthrough was the optimization of primary prevention strategy based on global vaccination campaigns, in combination with established measures to reduce person-to-person transmission. To date (July 2021), four vaccines have been 
approved by regulatory agencies in Europe: two mRNA vaccines encoding protein $\mathrm{S}$ and two adenoviral vector-based vaccines. The use of mRNA-vaccines (BNT162b2-PfizerBioNTech; mRNA-1273-Moderna) has spread rapidly worldwide because of the safety profile, the comparatively low cost of production, the capability of rapid development, and the higher efficacy [2].

Both national and international MS societies and expert opinions [3] encouraged vaccination for people with MS. Nevertheless, many people with MS (pwMS) are worried about COVID-19 vaccination, and a small but significant part of them (about 20\%) still refused it $[4,5]$. The main concern regards the possibility that disease-modifying therapies (DMTs) can negatively influence the safety and efficacy of COVID-19 vaccines.

Current evidence regarding the effect of DMTs on vaccine responses shows that some drugs can numb humoral immune responses to various vaccine types [6]. Particularly, pwMS taking cell-depleting agents (ocrelizumab, rituximab, ofatumumab, alemtuzumab, and cladribine) may probably have a milder vaccine response, especially if received during the maximum cell depletion period [7]. However, to date, only a few data about safety and efficacy of COVID19 vaccines in pwMS people are available. Chilimuri et al. published the first report of vaccine failure in a patient with relapsing-remitting MS on ocrelizumab that developed severe COVID-19, 19 days after receiving the last dose of the BNT162b2-Pfizer-BioNTech vaccine [8].

Achiron et al. evaluated the safety of the BNT162b2Pfizer-BioNTech COVID-19 vaccine in a cohort of MS patients in follow-up at MS center of Tel-Aviv (Israel) that received vaccination between December 2020 and January 2021 [9]. Five hundred fifty-five MS patients received the first dose of BNT162b2 vaccine, and 435 received the second dose. They reported three cases of SARS-CoV-2 infection after the first dose and no cases after the second dose. The safety profile of COVID-19 vaccine was similar to that observed in general population, and the incidence of relapses was similar to the rate in non-vaccinated patients.

The humoral response, mediated by B cell activation, provides a long-term immunologic memory and could be measured using the titer of antibodies against SARS-CoV-2 [10]. Although the significance of SARS-CoV2 antibodies testing is not yet clear, some evidence has suggested that they may have a protective role, together with $\mathrm{T}$ and $\mathrm{B}$ lymphocytes [11].

Achiron et al. tested the humoral immune response to the BNT162b2-Pfizer-BioNTech COVID-19 vaccine in a group of 125 pwMS (untreated or under treatment with fingolimod, cladribine, or ocrelizumab) compared to 47 healthy subjects similarly vaccinated [12]. They found that healthy controls, untreated MS patients, and MS patients under cladribine developed protective humoral immunity while only $22.7 \%$ of patients treated with ocrelizumab and $3.8 \%$ of patients treated with fingolimod demonstrated a protective humoral $\mathrm{IgG}$ response.

Since then, few case reports or small case series have confirmed these data, but, to our knowledge, no further prospective studies have been published so far about the safety and immunogenicity of COVID-19 vaccines in pwMS [13-16].

In our study conducted in a cohort of 140 PwMS, we tested immune response through $\mathrm{IgG}$ titer against SpikeRBD S1 protein, detected in a blood test performed about 30 days after the second vaccine injection. We also looked for a possible relationship between IgG production and different DMTs taken. Finally, we evaluated the adverse event profile related to SARS-CoV-2 mRNA vaccine administration.

\section{Methods}

\section{Study design and participants}

This is a multicentric, observational, prospective, cohort study conducted at three MS centers of Rome, Italy (Università Campus Bio-Medico, Fondazione Policlinico Universitario “A. Gemelli” IRCCS, and San Filippo Neri Hospital).

PwMS who were scheduled for the follow-up visit at their MS center between 26 April 2021 and 4 June 2021 were contacted by their neurologist and were informed about the study procedure and aims. In particular, the following inclusion criteria were considered: (1) diagnosis of MS according to the 2017 McDonald Criteria [17]; (2) complete COVID-19 vaccination (with BNT162b2-Pfizer-BioNTech or mRNA-1273-Moderna) completed in the previous month.

Patients who agreed to participate underwent the routine neurological visit 1 month after the second vaccine dose. After signing a written informed consent, we collected a blood sample to assess the titer of SARS-CoV-2 IgG antibodies. Subsequently, patients answered a questionnaire about eventual adverse events experienced after vaccination.

Demographics (age, gender), last laboratory findings (leukocyte and lymphocyte count, lymphocyte subpopulations), and clinical (MS type and duration, EDSS, DMTs exposure, medications) data were obtained from electronic health records of MS Centers.

All participants gave their written informed consent. This study was performed according to the declaration of Helsinki and was approved by the Local Ethics Committee.

\section{COVID-19 vaccination}

Vaccination against SARS-CoV-2 in Italy started at the end of 2020: frail patients, including pwMS, could receive the Pfizer-BioNTech COVID-19 (BNT162b2) vaccine. All 
patients received two doses ( $30 \mu \mathrm{g}, 0.3 \mathrm{~mL}$ each) administered intramuscularly, 3 weeks apart [18]. Then, the Moderna vaccine (mRNA-1273), similar in the biological mechanism to the Pfizer one, was also authorized among risk groups; two doses $(100 \mu \mathrm{g}, 0.5 \mathrm{~mL}$ each) are administered intramuscularly 28 days apart.

According to guideline recommendations of the Italian Society of Neurology (SIN) and Italian Association for Multiple Sclerosis (AISM) (https://www.aism.it/raccomandazioni_ aggiornate_sul_covid_19_le_persone_con_sclerosi_multipla_ sm\#vaccinazioni), all pwMS were recommended to be vaccinated against COVID-19 without stopping their current DMTs; patients treated with alemtuzumab, rituximab, and ocrelizumab were recommended to be vaccinated at least 12 weeks after DMTs administration or 4-6 weeks before DMT administration, if possible.

\section{Detection of SARS-CoV-2 IgG antibodies}

The detection of SARS-CoV-2 IgG antibodies in blood samples was performed using a chemiluminescent microparticle immunoassay for quantitative and qualitative detection of $\operatorname{IgG}$ against SARS-CoV-2 nucleoprotein (Spike-RBD S1) (SARSCoV-2 IgG II for use with ARCHITECT; Abbott Laboratories, Abbott Park, IL, USA; ref: 6S60-22). The assay was performed following the manufacturer's instructions, 1 month after the second dose. The humoral response was considered positive when the IgG titer was > 7.1 BAU/mL. All blood samples were analyzed at Università Campus Bio-Medico.

\section{Statistical analysis}

Continuous variables were described as mean \pm standard deviation unless otherwise specified. Dichotomic or categorical variables were expressed as frequencies. The relation between a positive SARS-CoV-2 IgG titer and MS therapy was explored with a chi-squared test while we used the Kruskal-Wallis test to evaluate eventual differences of the median SARS-CoV-2 IgG titer with respect to DMT exposure. In ocrelizumab patients, we assessed the relation between the SARS-CoV-2 IgG titer and the time from last DMT infusion through Pearson's correlation analysis. All two-tailed $p$-values $<0.05$ were considered significant. Data were analyzed using the Statistical Package for Social Sciences, version 22.0 (IBM SPSS, Inc., Chicago, Ill., USA).

\section{Results}

\section{Participants}

We enrolled 140 MS patients from 26 April 2021 and 4 June 2021. Baseline demographic and clinical characteristics are reported in Table 1. Women included in the cohort were 97 (69.3\%), and mean age was 43.5 years. Most of the patients had a relapsing disease course $(88.6 \%)$, and the median EDSS score was 2.0 (range 1.0-3.0). Only four patients had a previous history of COVID-19 infection (2.9\%). All but two patients received the BNT162b2 vaccine. Median time from the last vaccine dose to blood sampling was 31 days. Most patients (90\%) were in treatment with DMTs at the time of vaccination. Among patients not in treatment at the time of vaccination $(N=14), 9$ had never been treated with DMTs, 3 were treated with interferon (interrupted in 2006, 2008, and 2019, respectively), 1 was in treatment with glatiramer acetate until 2020, and 1 was in treatment with azathioprine until 2013.

Clinical and demographic data for each DMT group are reported in Table 2 . We do not find any statistically significant difference regarding sex and MS disease course. By contrast, patients on teriflunomide are older when compared to the other groups, and patients on second-line treatment (fingolimod, ocrelizumab, natalizumab, and alemtuzumab) have higher EDSS scores.

\section{Humoral immune response}

Four patients had a previous history of COVID-19 infection and were excluded for the following analysis since prior infection could have affected the antibody titer. At the time of vaccination, two patients were on treatment with glatiramer acetate, one with dimethyl fumarate, and the last one was treatment naïve. All of these patients had a positive humoral response.

One hundred eighteen (86.8\%) patients developed a positive humoral response (IgG titer $>7.1 \mathrm{BAU} / \mathrm{mL}$ ) against SARS-CoV-2, and the mean IgG titer was $1508 \mathrm{BAU} / \mathrm{mL}$ $( \pm 1627)$. Table 3 shows the humoral immune response after vaccination, based on the last DMT. Only some patients treated with fingolimod $(n=4,22.2 \%)$ and ocrelizumab $(n=14,66.0 \%)$ had a SARS-CoV-2 IgG titer below the limit of positivity ( $p<0.01$, chi-squared test). Moreover, patients treated with fingolimod and ocrelizumab had significantly lower IgG levels against SARS-CoV2 than the other groups $(p<0.01$, Kruskal-Wallis test; Fig. 1).

To better estimate the reduction of the humoral response in the fingolimod and the ocrelizumab groups, we put together all the patients from the untreated and the first-line DMTs (glatiramer acetate, interferons, dimethyl fumarate, teriflunomide) groups ( $n=66$ ), and we estimated the threshold of the lowest quartile (25th percentile; $\operatorname{IgG}$ titer $=746$ $\mathrm{BAU} / \mathrm{mL}$ ). None of the patients from the ocrelizumab group have a IgG titer against SARS-CoV-2 above this threshold, while three patients $(16.6 \%)$ of the fingolimod group have an IgG titer higher than $746 \mathrm{BAU} / \mathrm{mL}$. 
Table 1 Baseline characteristics

\begin{tabular}{ll}
\hline & MS patients \\
& $\boldsymbol{n}=\mathbf{1 4 0}$ \\
\hline Female sex, $n$ (\%) & $97(69.3)$ \\
Age, years & $43.5(12.7)$ \\
EDSS score, median [IQR] & $2.0[1.0-3.0]$ \\
MS course, $n(\%)$ & $124(88.6)$ \\
Relapsing & $16(11.4)$ \\
Progressive & \\
Previous SARS-CoV2 infection, $n(\%)$ & $4(2.9)$ \\
Vaccine type & $138(98.6)$ \\
Pfizer BNT162b2 & $2(1.4)$ \\
Moderna mRNA-1273 & \\
Time from vaccination to blood sample (days), median [IQR] & $31[29-35]$ \\
IgG anti-SARS-CoV2 titer (BAU/mL) & $1522.3(1616.3)$ \\
IgG anti-SARS-CoV2 titer (BAU/mL) after excluding patients with previous SARS-CoV2 infection & $1508.6(1627.4)$ \\
IgG titer above the positivity limit, $n$ (\%) & $122(87.1)$ \\
IgG titer above the positivity limit after excluding patients with previous SARS-CoV2 infection, $n(\%)$ & $118(86.8)$ \\
Any side effect, $n$ (\%) & $92(65.7)$ \\
DMTs, $n$ (\%) & $14(10.0)$ \\
No treatment & $11(7.9)$ \\
Glatiramer acetate & $7(5.0)$ \\
Inferferons & \\
Dimethyl fumarate & $23(16.4)$ \\
Teriflunomide & $11(7.9)$ \\
Fingolimod & $18(12.9)$ \\
Ocrelizumab & $25(17.9)$ \\
Natalizumab & $18(12.9)$ \\
Alemtuzumab & $10(7.1)$ \\
Azathioprine & $2(1.4)$ \\
\hline & $1(0.7)$ \\
\hline
\end{tabular}

All values are reported as mean (standard deviation) unless indicated otherwise

EDSS Expanded Disability Status Scale, IQR interquartile range, $M S$ multiple sclerosis, DMTs diseasemodifying therapies

In the ocrelizumab group, we found a significant positive correlation between the IgG levels and the time from last infusion to vaccination ( $r=0.44, p=0.026)$ (Fig. 2). The median time from the last ocrelizumab infusion to the first dose of vaccine was 4.9 months.

We did not find a significant correlation between total lymphocyte count (nor with lymphocyte subtypes including $\mathrm{CD} 4+, \mathrm{CD} 8+$, and CD19+ cells) and SARS-CoV-2 IgG titer in the ocrelizumab and the fingolimod groups.

Patients in treatment with alemtuzumab and cladribine had a very long-time interval from last DMT administration to vaccination (33.6 months for alemtuzumab and 12.5 for cladribine) with no patient with less than 6 months between last DMT exposure to vaccination.

\section{Safety}

Ninety-two patients (65.7\%) experienced at least one side effect after vaccination (Table 4). The most frequent was pain at the injection site $(57.1 \%)$, followed by fatigue (37.9\%), myalgia (27.1\%), fever (23.6\%), and headache (15.7\%). While local side effects (including pain, erythema, and swelling) had a similar incidence after both injections, systemic symptoms such as fatigue, fever, myalgia, and arthralgia were more frequent after the second vaccine administration. No patient experienced severe side effects requiring hospitalization or unusual adverse events.

\section{SARS-CoV-2 infection}

No SARS-CoV-2 infections were reported during the followup period (1 month after the second vaccination dose).

\section{Discussion}

This convenience sample of pwMS vaccinated against SARS-CoV-2 between April and June 2021 confirmed that mRNA vaccines are safe and well-tolerated and produced a protective humoral response in most of them (87.1\%).

We did not find any unusual side effect, and no patient reported a severe adverse event. Similarly to a previously described Israeli experience, our data showed that mRNA vaccines against SARS-CoV2 are generally well-tolerated in MS patients and that side effects are more frequent after the second dose, as for the general population [19].

For the first time, in our study, we evaluated the humoral response to mRNA vaccines against SARS-CoV2 
in a real-life cohort of MS patients that was extremely heterogeneous regarding the MS treatment, including both patients treated with first-line DMTs (such as interferon, glatiramer acetate, dimethyl fumarate, teriflunomide) and patients treated with high-efficacy DMTs (such as cladribine, ocrelizumab, alemtuzumab, natalizumab, and fingolimod). Indeed, previous studies have exclusively focused on patients treated with high-efficacy DMTs such as cladribine, ocrelizumab, fingolimod [12-16], natalizumab [20], and alemtuzumab [21].

Our data demonstrate that MS patients treated with oral or injectable first-line DMTs or natalizumab developed a measurable humoral response following the COVID-19 vaccine that was similar to untreated patients.

Moreover, in our cohort, all patients treated with alemtuzumab, azathioprine, and cladribine mounted an adequate humoral response to the vaccine. However, for all the patients in the alemtuzumab and cladribine groups, the time from last DMT administration to the vaccine was greater than 1 year. Therefore, our data could suggest that these drugs do not reduce the response to the vaccine after long-term exposure. Still, we cannot advise the minimum time between the last administration of these drugs and the vaccination. We have to acknowledge that the number of patients in treatment with these DMTs included in this study was very low, especially for azathioprine and cladribine ( 1 and 2 , respectively), thus preventing us from drawing definite conclusions.

Finally, we found that in some patients treated with ocrelizumab or fingolimod, the humoral immune response to the COVID-19 vaccine is impaired. Indeed, patients treated with these drugs had significantly lower IgG levels against SARS-CoV2 than the other groups, and some of them (22\% in the fingolimod group and $66 \%$ in the ocrelizumab group) failed to develop a significant humoral response. Our data confirm other reports including a large cohort from Israel and few case reports or small case series [12-16]. Moreover, the recently published data of the VELOCE trial showed a reduced humoral response to standard inactivated vaccines in MS patients treated with ocrelizumab [22]. The B-cell depletion, induced by ocrelizumab, could reduce the production of neutralizing antibodies against SAR-CoV-2 that prevent the virus from entering the cell or lyse the infected cells. This might explain the observations where ocrelizumab was associated with more severe forms of COVID-19 [23, 24]

Taken together, these findings demonstrate that ocrelizumab and fingolimod can negatively influence the humoral response to COVID-19 vaccination, thus suggesting a limited efficacy in vaccinating pwMS in treatment with such DMTs.

However, some aspects should be considered before using these data to change the current guidelines that recommend 
Table 3 Vaccine response based on DMTs exposure

\begin{tabular}{|c|c|c|c|c|}
\hline & IgG anti-SARS-CoV2 titer & $\begin{array}{l}\text { IgG titer above the positivity } \\
\text { limit, } n(\%)\end{array}$ & $\begin{array}{l}\text { Any side effect, } \\
n(\%)\end{array}$ & $\begin{array}{l}\text { Time from last drug infusion/intake } \\
\text { to vaccination (months) }\end{array}$ \\
\hline No treatment (14) & 1359 [736-3130] & $13(100)$ & $11(78.6)$ & NA \\
\hline Glatiramer acetate (11) & 931 [706-1877] & $9(100)$ & $7(63.6)$ & NA \\
\hline Interferons (7) & $2858[1890-3814]$ & $7(100)$ & $7(100)$ & NA \\
\hline Dimethyl fumarate (23) & 1931 [743-3860] & $22(100)$ & $18(78.3)$ & NA \\
\hline Teriflunomide (11) & 968 [306-2710] & $11(100)$ & $8(72.7)$ & NA \\
\hline Fingolimod (18) & $53[8-168]^{*}$ & $14(77.8)^{*}$ & $8(44.4)$ & NA \\
\hline Ocrelizumab (25) & $5[1-20]^{*}$ & $11(44.0)^{*}$ & $12(48.0)$ & $4.9(1.4)$ \\
\hline Natalizumab (18) & 2022 [1012-3728] & $18(100)$ & $15(83.3)$ & $0.6(0.3)$ \\
\hline Alemtuzumab (10) & 2133 [1338-3127] & $10(100)$ & $5(50)$ & $33.6(11.7)$ \\
\hline Cladribine (2) & 1041 & $2(100)$ & $1(50)$ & $12.5(10.9)$ \\
\hline Azathioprine (1) & 2426 & $1(100)$ & $0(0)$ & NA \\
\hline
\end{tabular}

All values are reported as mean (standard deviation) unless indicated otherwise

We excluded patients with previous infection from "IgG anti-SARS-CoV2 titer" and "IgG titer above the positivity limit" columns $D M T s$ disease-modifying therapies

* $p<0.01$

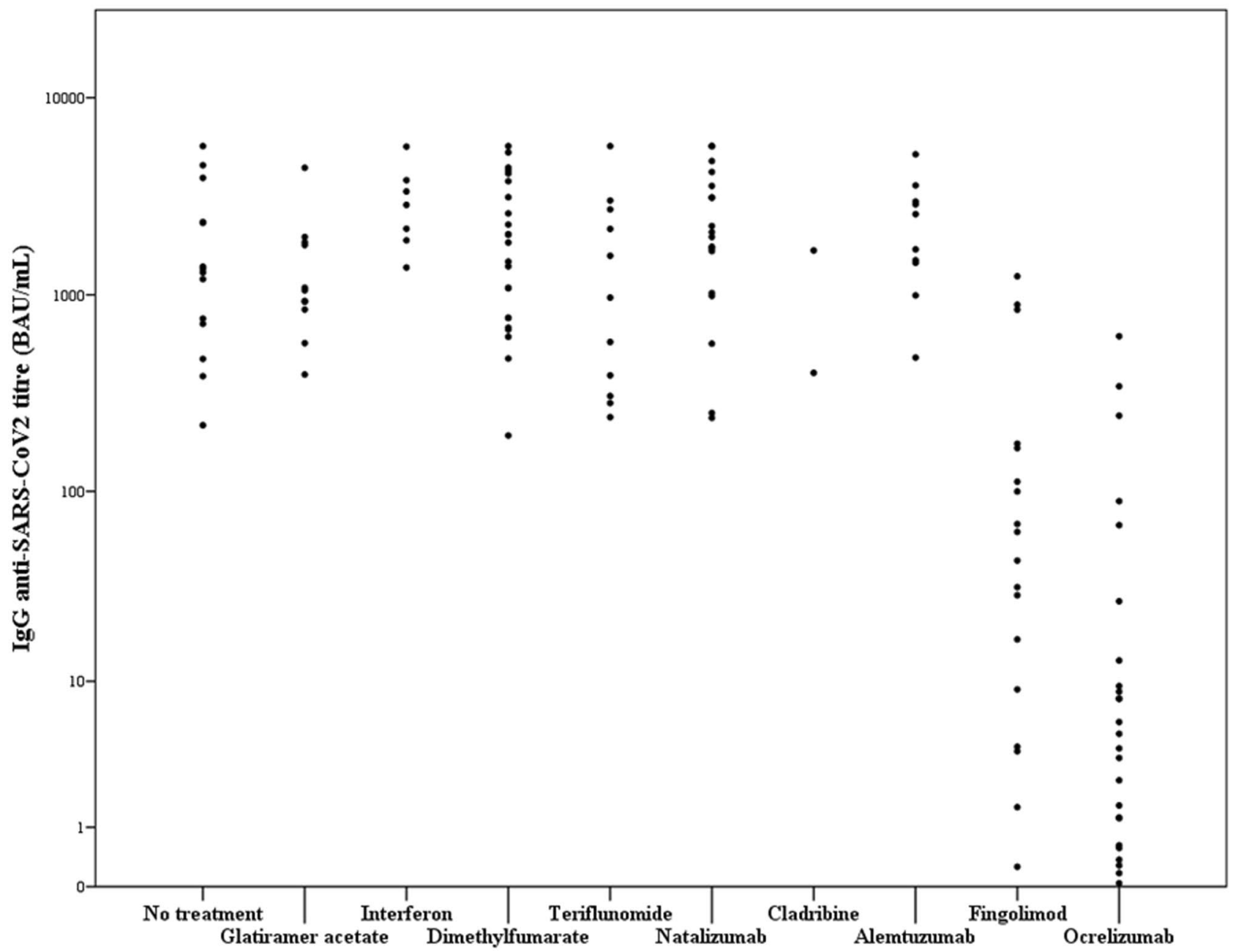

Fig. 1 Post-vaccination SARS-CoV-2 IgG antibody titer by DMTs. The horizontal line represents the positivity limit for a positive humoral response (IgG titer $>7.1 \mathrm{BAU} / \mathrm{mL})$ 


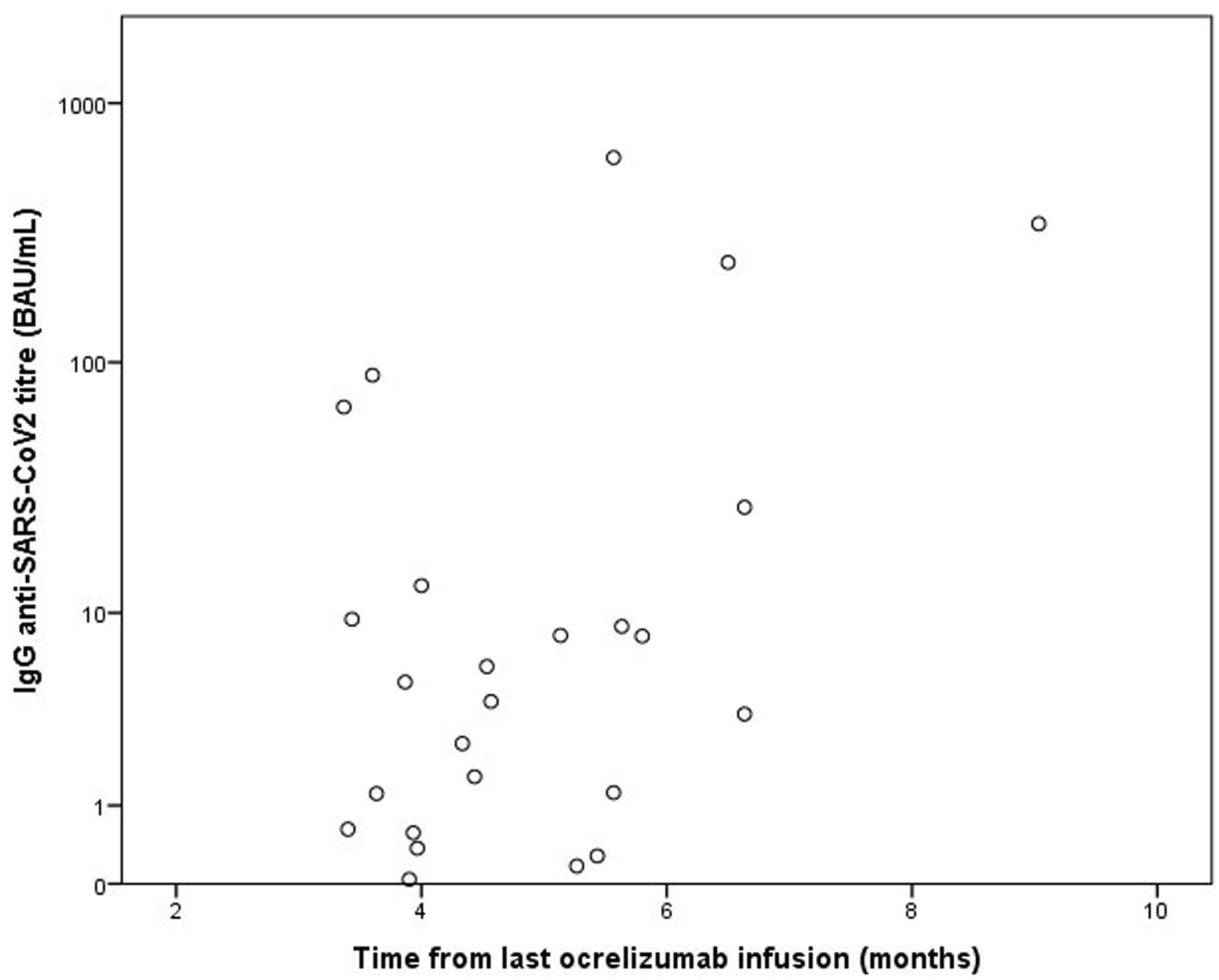

Fig. 2 Correlation between SARS-CoV-2 IgG titer and time from last ocrelizumab infusion to vaccination. We find a significant positive correlation between those two variables $(r=0.44, \mathrm{p}=0.026)$. SARS-CoV-2 IgG titer is reported with a logarithmic scale

vaccination to all people with MS regardless of their current DMTs.

First, according to available data, the humoral response among pwMS receiving these drugs is extremely variable. For instance, in our cohort, the proportion of patients in treatment with fingolimod or ocrelizumab who developed a protective IgG titer following COVID-19 vaccination was higher than reported from Achiron et al. (78\% vs 3.8\% for fingolimod, $34 \%$ vs $22.7 \%$ for ocrelizumab) [12]. Guerrieri et al. also reported similar results $(62.5 \%$ for fingolimod and $37.5 \%$ for ocrelizumab) [13]. This difference could be explained, at least in part, by the different sensitivity of the methods used for antibody detection [25].

Secondly, although some patients exposed to ocrelizumab or fingolimod mounted a reduced humoral response, we cannot exclude the possibility of an adequate and protective immunological cell-mediated response to vaccination as suggested by a few case reports, and further studies are ongoing to clarify this aspect [26].

Our data demonstrated a positive and significant correlation between the antibody titer and the time from last ocrelizumab infusion to vaccination, thus suggesting that an extended interval dosing protocol could improve vaccine performance in these patients. Moreover, recent studies found that an extended interval dosing protocol does not reduce the effectiveness of ocrelizumab treatment [27].

Finally, it has been recently demonstrated that in immunosuppressed patients for solid-organ transplantation, the administration of a third dose of the BNT162b2 vaccine significantly improves the immunogenicity of the vaccine [28]. As of this writing, no data have been published for pwMS, but the administration of additional doses of COVID-19 vaccine in patients in treatment with fingolimod or ocrelizumab could be considered. 
Table 4 Side effects

\begin{tabular}{llll}
\hline & $\begin{array}{l}\text { Any vaccine } \\
\text { administration, } \\
n(\%)\end{array}$ & $\begin{array}{l}\text { First } \\
\text { vaccine } \\
\text { dose, } n(\%)\end{array}$ & $\begin{array}{l}\text { Second } \\
\text { vaccine dose, } \\
n(\%)\end{array}$ \\
\hline Any side effect & $92(65.7)$ & $82(58.6)$ & $87(62.0)$ \\
Pain on injection site & $80(57.1)$ & $72(51.4)$ & $62(44.3)$ \\
Skin erythema & $15(10.7)$ & $10(7.1)$ & $9(6.4)$ \\
Swelling & $14(10.0)$ & $14(10.0)$ & $10(7.1)$ \\
Fatigue & $53(37.9)$ & $35(25.0)$ & $45(32.1)$ \\
Headache & $31(22.1)$ & $22(15.7)$ & $21(15.0)$ \\
Myalgia & $38(27.1)$ & $21(15.0)$ & $28(20.0)$ \\
Arthralgia & $22(15.7)$ & $12(8.6)$ & $20(14.3)$ \\
Fever & $33(23.6)$ & $10(7.1)$ & $29(20.7)$ \\
Adenopathy & $4(2.9)$ & $4(2.9)$ & $4(2.9)$ \\
Urticaria & $1(0.1)$ & $1(0.1)$ & $1(0.1)$ \\
\hline
\end{tabular}

Our study has some limitations. First, the sample is relatively small, and the number of patients in each medication group is relatively low (especially for cladribine and azathioprine). Second, we did not assess the immunological cell-mediated response to vaccination that could significantly contribute to anti-SARS-CoV-2 immunity, especially in patients that did not develop a humoral response. Moreover, we did not specifically measure the titers of neutralizing antibodies. Even if the serology test used in our study is highly correlated to neutralization activity [29], we do not know to what extent patients' $\mathrm{IgG}$ levels detected in the sera actually confer a protective humoral response. Finally, we did not require pre-vaccination serological tests for participants. Although we recorded in our database COVID-19 cases, we cannot exclude a previous asymptomatic infection that could influence the serological response.

The most important strengths of our study are the prospective and multicentric design with the antibody detection that was centralized and performed at fixed time points using the same methodology for all patients.

\section{Conclusions}

Our study confirms that COVID-19 vaccination is safe and well-tolerated in pwMS and should be recommended to all patients regardless of their current DMTs. Indeed, according to our data, patients in treatment with oral or injectable first-line DMTs, natalizumab, cladribine, and alemtuzumab develop a measurable humoral response. Some patients in treatment with fingolimod and ocrelizumab could have a reduced humoral response. Accordingly, in patients treated with these drugs, the detection of SARS-CoV-2 IgG antibodies following vaccination and the administration of a third dose of vaccine could be considered. Further studies are required to confirm these results in larger samples, to clarify the role of cell-mediated immunity in vaccine response.
Supplementary Information The online version contains supplementary material available at https://doi.org/10.1007/s13311-021-01165-9.

Required Author Forms Disclosure forms provided by the authors are available with the online version of this article.

\section{References}

1. [https://covid19.who.int] accessed on 18 Aug 2021.

2. Ahammad I, Lira SS. Designing a novel mRNA vaccine against SARS-CoV-2: an immunoinformatics approach. Int J Biol Macromol. 2020;162:820-37. https://doi.org/10.1016/j.ijbiomac. 2020.06.213.

3. Centonze D, Rocca MA, Gasperini C, Kappos L, Hartung HP, Magyari M, et al. Disease-modifying therapies and SARS$\mathrm{CoV}-2$ vaccination in multiple sclerosis: an expert consensus. J Neurol 2021;1-8. https://doi.org/10.1007/s00415-021-10545-2.

4. Ehde DM, Roberts MK, Herring TE, Alschuler KN. Willingness to obtain COVID-19 vaccination in adults with multiple sclerosis in the United States. Multiple sclerosis and related disorders. 2021;49: 102788. https://doi.org/10.1016/j.msard.2021.102788.

5. Serrazina F, Pinho AS, Cabral G, Salavisa M, Correia AS Willingness to be vaccinated against COVID-19:an exploratory online survey in a Portuguese cohort of multiple sclerosis patients. Multiple sclerosis and related disorders. 2021;51: 102880. https://doi.org/10.1016/j.msard.2021.102880.

6. Ciotti JR, Valtcheva MV, Cross AH. Effects of MS disease-modifying therapies on responses to vaccinations: a review. Multiple sclerosis and related disorders. 2020;45: 102439. https://doi.org/10.1016/j.msard. 2020.102439.

7. Kelly H, Sokola B, Abboud H. Safety and efficacy of COVID-19 vaccines in multiple sclerosis patients. J Neuroimmunol. 2021;356: 577599. https://doi.org/10.1016/j.jneuroim.2021.577599.

8. Chilimuri S, Mantri N, Gongati S, Zahid M, Sun H. COVID-19 vaccine failure in a patient with multiple sclerosis on ocrelizumab. Vaccines. 2021;9(3). https://doi.org/10.3390/vaccines9030219.

9. Achiron A, Dolev M, Menascu S, Zohar DN, Dreyer-Alster S, Miron S, et al. COVID-19 vaccination in patients with multiple sclerosis: what we have learnt by February 2021. Multiple sclerosis (Houndmills, Basingstoke, England). 2021;27(6):864-70. https://doi.org/10.1177/13524585211003476.

10. Barnes CO, Jette CA, Abernathy ME, Dam KA, Esswein SR, Gristick HB, et al. SARS-CoV-2 neutralizing antibody structures inform therapeutic strategies. Nature. 2020;588(7839):682-7. https://doi.org/10.1038/s41586-020-2852-1.

11. Khoury DS, Cromer D, Reynaldi A, Schlub T, Wheatley A, Juno J, et al. Neutralizing antibody levels are highly predictive of immune protection from symptomatic SARS-CoV-2 infection. Nat Med. 2021;27(7):1205-11. https://doi.org/10.1038/ s41591-021-01377-8.

12. Achiron A, Mandel M, Dreyer-Alster S, Harari G, Magalashvili D, Sonis P, et al. Humoral immune response to COVID-19 mRNA vaccine in patients with multiple sclerosis treated with high-efficacy disease-modifying therapies. Ther Adv Neurol Disord. 2021;14:17562864211012836. https://doi.org/10.1177/ 17562864211012835 .

13. Guerrieri S, Lazzarin S, Zanetta C, Nozzolillo A, Filippi M, Moiola L. Serological response to SARS-CoV-2 vaccination in multiple sclerosis patients treated with fingolimod or ocrelizumab: an initial real-life experience. J Neurol. 2021:1-5. https://doi.org/ 10.1007/s00415-021-10663-x.

14. Bigaut K, Kremer L, Fleury M, Lanotte L, Collongues N, de Seze J. Impact of disease-modifying treatments on humoral response 
after COVID-19 vaccination: a mirror of the response after SARSCoV-2 infection. Revue neurologique. 2021. https://doi.org/10. 1016/j.neurol.2021.05.001.

15. Buttari F, Bruno A, Dolcetti E, Azzolini F, Bellantonio P, Centonze $\mathrm{D}$, et al. COVID-19 vaccines in multiple sclerosis treated with cladribine or ocrelizumab. Multiple sclerosis and related disorders. 2021;52: 102983. https://doi.org/10.1016/j.msard.2021.102983.

16. Gallo A, Capuano R, Donnarumma G, Bisecco A, Grimaldi E, Conte $\mathrm{M}$, et al. Preliminary evidence of blunted humoral response to SARS-CoV-2 mRNA vaccine in multiple sclerosis patients treated with ocrelizumab. Neurological Sciences : Official Journal of the Italian Neurological Society and of the Italian Society of Clinical Neurophysiology. 2021;1-4. https://doi.org/10.1007/ s10072-021-05397-7.

17. Thompson AJ, Banwell BL, Barkhof F, Carroll WM, Coetzee T, Comi G, et al. Diagnosis of multiple sclerosis: 2017 revisions of the McDonald criteria. The Lancet Neurology. 2018;17(2):16273. https://doi.org/10.1016/s1474-4422(17)30470-2.

18. Oliver SE, Gargano JW, Marin M, Wallace M, Curran KG, Chamberland $\mathrm{M}$, et al. The Advisory Committee on Immunization Practices' Interim Recommendation for Use of Pfizer-BioNTech COVID-19 Vaccine - United States, December 2020. MMWR Morb Mortal Wkly Rep. 2020;69(50):1922-4. https://doi.org/10. 15585/mmwr.mm6950e2.

19. Lotan I, Wilf-Yarkoni A, Friedman Y, Stiebel-Kalish H, Steiner I, Hellmann MA. Safety of the BNT162b2 COVID-19 vaccine in multiple sclerosis (MS): early experience from a tertiary MS center in Israel. Eur J Neurol. 2021. https://doi.org/10.1111/ene. 15028.

20. Capuano R, Donnarumma G, Bisecco A, Grimaldi E, Conte M, d'Ambrosio A, et al. Humoral response to SARS-CoV-2 mRNA vaccine in patients with multiple sclerosis treated with natalizumab. Ther Adv Neurol Disord. 2021;14:17562864211038112. https://doi.org/10.1177/17562864211038111.

21. Drulovic J, Ivanovic J, Martinovic V, Tamas O, Veselinovic N, Cujic D, et al. Humoral response to SARS-CoV-2 COVID-19 vaccines in patients with multiple sclerosis treated with immune reconstitution therapies. Multiple sclerosis and related disorders. 2021;54: 103150. https://doi.org/10.1016/j.msard.2021.103150.
22. Bar-Or A, Calkwood JC, Chognot C, Evershed J, Fox EJ, Herman A, et al. Effect of ocrelizumab on vaccine responses in patients with multiple sclerosis: the VELOCE study. Neurology. 2020;95(14):e1999-2008. https://doi.org/10.1212/wnl. 0000000000010380 .

23. Sormani MP, Salvetti M, Labauge P, Schiavetti I, Zephir H, Carmisciano L, et al. DMTs and Covid-19 severity in MS: a pooled analysis from Italy and France. 2021;8(8):1738-44. https://doi.org/ 10.1002/acn3.51408.

24. Sormani MP, De Rossi N, Schiavetti I, Carmisciano L, Cordioli $\mathrm{C}$, Moiola L, et al. Disease-modifying therapies and coronavirus disease 2019 severity in multiple sclerosis. 2021;89(4):780-9. https://doi.org/10.1002/ana.26028.

25. Harritsh $\varnothing \mathrm{j}$ LH, Gybel-Brask M, Afzal S, Kamstrup PR, Jørgensen CS, Thomsen MK, et al. Comparison of 16 serological SARSCoV-2 immunoassays in 16 clinical laboratories. J Clin Microbiol. 2021;59(5). https://doi.org/10.1128/jcm.02596-20.

26. Ferguson J, Murugesan K, Banaei N, Liu A. Interferon-gamma release assay testing to assess COVID-19 vaccination response in a SARS-CoV-2 seronegative patient on rituximab: a case report. International journal of infectious diseases : IJID : official publication of the International Society for Infectious Diseases. 2021. https://doi.org/10.1016/j.ijid.2021.06.054.

27. Rolfes L, Pawlitzki M, Pfeuffer S, Nelke C, Lux A, Pul R, et al. Ocrelizumab extended interval dosing in multiple sclerosis in times of COVID-19. Neurology(R) neuroimmunology \& neuroinflammation. 2021;8(5). https://doi.org/10.1212/nxi.0000000000001035.

28. Kamar N, Abravanel F, Marion O, Couat C, Izopet J, Del Bello A. Three doses of an mRNA Covid-19 vaccine in solid-organ transplant recipients. N Engl J Med. 2021;385(7):661-2. https:// doi.org/10.1056/NEJMc2108861.

29. Jung K, Shin S, Nam M, Hong YJ, Roh EY, Park KU, et al. Performance evaluation of three automated quantitative immunoassays and their correlation with a surrogate virus neutralization test in coronavirus disease 19 patients and pre-pandemic controls. J Clin Lab Anal. 2021;35(9): e23921. https://doi.org/10.1002/jcla.23921.

Publisher's Note Springer Nature remains neutral with regard to jurisdictional claims in published maps and institutional affiliations. 